\title{
Hybrid allele-specific ChIP-Seq analysis links variation in transcription factor binding to traits in maize
}

Thomas Hartwig ( $\nabla$ thartwig@mpipz.mpg.de)

Heinrich Heine University Düsseldorf https://orcid.org/0000-0002-2707-2771

Michael Banf

Carnegie Institution for Science

Gisele Prietsch

Carnegie Institution for Science

Julia Engelhorn

Heinrich Heine Universit\&\#x00E4

Jinliang Yang

University of Nebraska-Lincoln https://orcid.org/0000-0002-0999-3518

Zhi-Yong Wang

Carnegie Institution for Science

\section{Research Article}

Keywords: maize, ChIP-Seq, transcription factor binding

Posted Date: June 28th, 2021

DOI: https://doi.org/10.21203/rs.3.rs-543958/v1

License: (9) (i) This work is licensed under a Creative Commons Attribution 4.0 International License.

Read Full License 


\section{Hybrid allele-specific ChIP-Seq analysis links variation in transcription factor binding to traits in maize}

Thomas Hartwig ${ }^{1,2,3^{*}}$, Michael Banf ${ }^{1,3}$, Gisele Passaia Prietsch ${ }^{1}$, Julia Engelhorn²,3, Jinliang Yang ${ }^{4}$; ZhiYong Wang ${ }^{1 *}$

${ }^{1}$ Carnegie Institution for Science, 260 Panama Street, Stanford CA 94305, USA

${ }^{2}$ Heinrich-Heine University, Universitätsstraße 1, Düsseldorf NRW 40225, Germany

${ }^{3}$ Max Planck Institute for Plant Breeding Research, Carl-von-Linné-Weg 10, Cologne NRW 50829, Germany

${ }^{4}$ University of Nebraska-Lincoln, Lincoln, 363 Keim Hall, NE 68583, USA

* Author for correspondence: thartwig@mpipz.mpg.de; zywang24@stanford.edu 
Introductory paragraph

Variation in transcriptional regulation is a major cause of phenotypic diversity ${ }^{1}$. Genomewide association studies (GWAS) have shown that most functional variants reside in noncoding regions, where they potentially affect transcription factor (TF) binding and chromatin accessibility to alter gene expression. Pinpointing such regulatory variations, however, remains challenging ${ }^{2}$. Here, we developed a hybrid allele-specific chromatin binding sequencing (HASCh-seq) approach and identified variations in target binding of the brassinosteroid (BR) responsive transcription factor ZmBZR1 in maize. Chromatin immunoprecipitation followed by sequencing (ChIP-seq) in B73xMo17 F1s identified thousands of target genes of ZmBZR1. Allele-specific ZmBZR1 binding (ASB) was observed for about $14.3 \%$ of target genes. It correlated with over 550 loci containing sequence variation in BZR1-binding motifs and over 340 loci with haplotype-specific DNA methylation, linking genetic and epigenetic variations to ZmBZR1 occupancy. Comparison with GWAS data linked hundreds of ASB loci to important yield, growth and disease-related traits. Our study provides a robust method for analyzing genome-wide variations of transcription factor occupancy and identified genetic and epigenetic variations of the $B R$ response transcription network in maize.

$\mathrm{BR}$ is a major growth-promoting hormone that controls many important agronomic traits, such as plant height and architecture, shoot branching, flowering time, sex determination, seed size, and disease resistance ${ }^{3-5}$. Studies in Arabidopsis have shown that BR binds to the receptor kinase BRI1 to activate the BZR1 family of TFs which directly regulate thousands of target genes involved in various cellular and developmental responses ${ }^{6}$. 
Genetic evidence indicates that components of the BR signaling pathway, including BZR1, are conserved in monocotyledonous crops such as rice and maize ${ }^{5,7,8}$. Given the severe and broad developmental phenotypes of BR deficient and signaling mutants, we expect that evolutionary variations in the BR transcriptional targets would be a major contributor to phenotypic diversity 5,9 .

To characterize the BR-regulated transcriptional network in maize, we performed RNA-seq on shoot tissue of wild type as well as BR biosynthesis deficient brd1 (BR6ox2) seedlings treated with and without brassinolide (BL), the most active BR. We identified a total of 2743 BR-responsive genes (Supplementary Table 1). To identify the genes directly regulated by the BR signaling pathway in vivo, we performed ChIP-seq analysis of the maize BES1/BZR1 homolog (Zm00001d021927), using transgenic plants (B73 backcrossed) expressing a ZmBES1/BZR1-YFP fusion protein driven by the ZmBES1/BZR1 promoter described previously ${ }^{10}$. The nuclear localization of ZmBES1/BZR1-YFP was increased by BR and reduced by RNAi silencing of BRI1 ${ }^{10}$, and by the BR biosynthesis inhibitor propiconazole (PPZ ${ }^{11,12}$ ) (Supplementary Fig. 1). Such BR-dependent nuclear localization is similar to that observed for BZR1 in Arabidopsis, rice, and tomato ${ }^{13-16}$, suggesting that ZmBZR1 plays a conserved role in BR-responsive gene regulation.

Our ChIP-seq experiment identified 17463 high confidence ZmBZR1 binding peaks (Supplementary Table 2), most of which were near the transcription start site (Fig. 1a). The list of potential target genes included maize homologs of known AtBZR1 targets, such as ZmBR6ox2 and ZmIAA19 (Fig. 1b, Supplementary Fig. 1) ${ }^{6}$, some of which were validated by ChIP-qPCR (Supplementary Fig. 1). Our analysis of cis-element enrichment 
identified the BR response element (BRRE, CGTG(C/T)G) and the G-box (CACGTG), which were previously identified as BZR1 binding sites in Arabidopsis ${ }^{6,17}$ (Fig. 1C). Interestingly, co-enrichment of the binding sites for TCP factors (e.g. GGC/ACCA) was also observed, similar to the observation in Arabidopsis ${ }^{6}$ (Fig. 1d). These results indicate that the BR response cis-elements and their combination with other factors are conserved in Arabidopsis and maize.

The ZmBZR1 ChIP-seq peaks located near 6371 putative target genes (Supplementary Table 3), which included (according to the RNA-seq analysis) 580 and 469 BR activated and repressed genes, respectively (Fig. 1e). Overall, maize BZR1 target genes had 2058 unique Arabidopsis annotated orthologs, of which 519 (25.2\%) were previously identified as AtBZR1 targets $^{18}$ (Fig. 1f, Supplementary Table 4, Supplementary Fig. 1). Among orthologous genes between the two species, we found the highest enrichment over random for BR-responsive BZR1 targets compared to e.g. non-responsive targets or BRresponsive non-target genes (Fig. 1g). The conserved BR-responsive target genes tended to show the same direction of response (Fig. 1h). The results indicate both conservation and evolution among the transcriptional targets of BR signaling.

Although it is poorly understood how non-coding variants affect phenotypes, nearly half of quantitative trait variation in maize is explained by accessible, non-coding regions that may contain regulatory elements, e.g. TF binding sites ${ }^{2,19}$. While molecular approaches such as ChIP-seq can identify TF binding sites, quantitative analyses of the effects of genetic and epigenetic variations on TF binding often suffer from sample-tosample variations and trans-factor variations. To address these challenges, we performed ChIP-seq in F1 hybrids and named the approach HASCh-seq. To understand the 
functions of BZR1 binding in regulating gene expression and plant traits, we studied the influence of genetic variation between two inbred lines, B73 and Mo17, on BZR1 binding (Fig. 2a). Single nucleotide polymorphisms (SNPs) that affect, or link to, BZR1 binding will show a shift of the allele frequency from the expected 1:1 ratio in an F1 (Fig. 2b). We observed a strong reproducibility between the biological replicates (3x B73xMo17 and 3x Mo17xB73; Pearson's correlation coefficient $>$ 0.88, Supplementary Fig. 1). Combined analysis of the ChIP-seq results identified 29902 high-confidence BZR1 binding peaks (Supplementary Table 5) flanking 9490 genes (Supplementary Table 6) in the hybrids, and 19550 SNPs that showed a significant allelic bias $(p<0.001)$. We further focused our analysis on 8335 of those SNPs located within high-confidence BZR1-binding peaks (Supplementary Table 7) and within 3297 linkage groups (Supplementary Table 8, Fig. 2c). The lead SNP of each linkage group (i.e. showing the most significant bias) was considered as putative allele-specific BZR1 binding sites (ASBs).

About $19.2 \%$ of ASBs were within 1 -kb upstream of the transcription start sites of genes. About $56.8 \%$ of ASBs were found within $5 \mathrm{~kb}$ upstream and $1 \mathrm{~kb}$ downstream of 1355 genes, which are considered putative BZR1 ASB target genes (Supplementary Table 9, Fig. 2d). An analysis of the higher affinity allele sequence of ASB regions revealed BZR1-binding motifs (BRRE and G-box) as the most enriched (Fig. 2e). Of the BZR1-binding motifs that overlapped with ASB SNPs (i.e. were altered compared to the canonical motif), a decreased BZR1 binding was observed for $87.5 \%$ and $94.3 \%$ of variations in BRRE and of G-box motifs, respectively (Fig. 2f). A 2.1-fold higher frequency of variations affecting BZR1 binding was observed for the core "CGTG" sequence than the two flanking bases (Fig. 2g), consistent with results of a previous mutagenesis study 
showing the importance of this core sequence for BZR1 binding ${ }^{17}$. These results indicate that variations in the binding site sequences affect BZR1 occupancy in vivo.

Variation in canonical BZR1 binding motifs accounted for $554(16.8 \%)$ of all ASBs (Fig. 2h). It is possible that SNPs outside the canonical cis-elements affect BZR1 occupancy, by directly affecting the DNA binding of BZR1 or its heterodimer partner $\mathrm{TFs}^{18,20,21}$. Alternatively, haplotype specific DNA methylation or histone modification may affect TF binding ${ }^{22-24}$ and contribute to allele-specific BZR1 occupancy. Therefore, we compared our BZR1 ASB data with genome-wide DNA methylation data of B73 and Mo1722. Based on observation in recombinant inbred and hybrid lines of B73/Mo17, over $90 \%$ of the parental methylation status is preserved ${ }^{22,23}$. We found significant $(p<0.05$, hypergeometric test) allele-specific variation in DNA methylation for a large fraction of ASBs (346, 10.6\%) including 53 (15.3\%) ASBs that also overlapped with canonical BZR1 motifs (Fig. 2h, Supplementary Table 10). Further, there were strong correlations between reduced BZR1 binding and hypermethylation at both CG or CHG sites (Fig. 2i-j), which are known to be associated with repression of transcription ${ }^{22}$. In contrast, $\mathrm{CHH}$ methylation accumulated in the regions flanking BZR1 binding instead of directly overlapping with ASBs (Fig. 2k). This is consistent with the finding that $\mathrm{CHH}$ methylation occurs de novo in DNA upstream and downstream of highly expressed genes ${ }^{24}$. For the majority of ASBs (89.5\%), both alleles were hypomethylated. Among the differentially methylated loci, $96.5 \%$ (334/346) showed BZR1 binding bias towards the hypomethylated allele of at least 6.6 fold (Fig. 2I, Supplementary Fig. 2, Supplementary Table 10). These results indicate that $\mathrm{CG}$ and $\mathrm{CHG}$ methylation drastically reduce BZR1 occupancy. 
To determine whether variations of BZR1 binding contribute to differential gene expression, we compared our ASB data with the data of allele-specific transcriptome analysis in the B73xMo17 hybrid. About $24.6 \%$ of all maize genes showed allele-specific differential expression in B73xMo17 hybrids (Fig. 3a) $)^{25}$, while $38.1 \%$ (433 of the 1137) ASB-associated genes showed allele-specific variation in gene expression (1.55-fold enrichment, $p=1.1^{*} 10^{-24}$ assuming a hypergeometric distribution, Fig. $\left.3 b\right)$. This suggests that variation of BZR1 binding may contribute to variation of gene expression in the hybrid. Indeed, RT-qPCR analysis of BR responsive expression of ASB-associated BZR1 target genes, in B73 and Mo17 plants, confirmed that stronger BZR1 binding correlated with higher BR responsiveness of both BR-induced, VP14 ${ }^{26}$, and BR repressed, DWF427, target genes in the inbred lines treated with or without PPZ and brassinolide (Fig. 3c$3 h)^{11}$.

In addition to cis-regulatory elements located in the close vicinity to the transcriptin start site (TSS) or transcription termination site (TTS), transcriptional enhancers can be located tens of kilobases from their target genes ${ }^{28}$. We thus hypothesized that the ASBs located in intergenic regions could be located within distant enhancers and analyzed their abundance around putative intergenic enhancer regions ${ }^{29}$. Of the ASBs distant from the genes, $11 \%$ coincided with the 1495 intergenic enhancer regions identified in B73, which is approximately 37 -fold higher than random probability (Fig. 4a).

To assess the relationship between variations of BZR1 binding and trait variations, we quantified the enrichment of GWAS hits across 41 traits $^{2}$ within $2 \mathrm{~kb}$ of ASB regions. We found a 2.2-5.5 fold enrichment for 16 of the traits, compared to background SNPs with similar genomic distribution and minor allele frequency (bgSNPs, see methods). 
While the largest fraction $(40 \%, 1.5$ fold enrichment within the dataset) were traits related to growth and yield, other known phenotypic variations between B73 and Mo17 such as tassel branching ${ }^{30}$ and disease resistance ${ }^{31}$ were also enriched (Fig 4b). To further investigate the role of ASBs in complex organismal trait variation we used variance component annotation (VCAP). We partitioned the heritable phenotypic variance into annotation-specific components classifying ASB and bgSNP regions ${ }^{19}$. We examined the Nested Association Mapping (NAM) population using VCAP of ASBs and found that they explained a remarkable portion of the heritable variance (>10\%) for some of the traits with moderate to high heritability (h2 $>0.4 \mathrm{l}$ ). For 16 of the 25 traits analyzed, we found that ASBs explained disproportionately larger genetic variances compared to the bgSNPs, including 100 kernel weight, tassel branch number, and northern leaf blight resistance, but not e.g. the ratio of ear height to total plant height (Fig. 4c).

In summary, we present HASCh-seq as a robust method for identifying genetic variants that affect TF binding. By analyzing the TF binding to two different alleles in the same cells, HASCh-seq avoids technical variations that compromises quantitation and trans-factor differences that complicate data interpretation. Our HASCh-seq analysis of ZmBZR1 demonstrates a high level of variations in the BR transcriptional regulatory network between two diverse maize inbreds. Differences of ZmBZR1 occupancy were correlated with variations in its binding motif sequences and DNA methylation status. Our data also provides genetic evidence for functions of thousands of cis-elements in the BR transcription network in maize. The approach complements classical GWAS approaches, as there were significant associations between ASBs and BR-regulated traits ${ }^{32}$. This 
demonstrates that combining GWAS with HASCh-seq can be a powerful approach to pinpoint candidate targets for genome editing to improve traits. 


\section{Acknowledgement}

We are grateful to A. Luo and A. W. Sylvester for providing the transformed BES1/BZR1YFP maize lines. We are grateful to Marty Sachs for helpful communications concerning maize mutants and the Maize Genetics Cooperation Stock Center for providing the brd1 mutant seeds. We thank M. Evans, Y. Lu, D. Sosso and V. Walbot for maize field support. We thank S. E. Castel for the merge-sam script. We thank G.W. Huntress and M. Lopez for IT support and the Stanford genomic center for sequencing support. We are grateful to H. Cartwright for confocal microscopy. We also thank W. B. Frommer, S. Rhee, R. Weizbauer, and C. H. Park for their feedback and critical discussion of the paper. This work was supported by a grant from NIH (R01GM066258) to Z. Y. W.

\section{Author Contributions}

Z.Y.W conceived the research project. T.H., J.Y., G.P., and Z.Y.W. designed experiments. T.H., G.P., and J.Y. performed experiments. T.H., M.B., G.P., J.E., J.Y., and Z.Y.W. analyzed the data. T.H., J.E., and Z.Y.W. wrote the manuscript, and G.P., J.Y., and M.B. revised it.

\section{Data availability}

The data discussed in this publication has been deposited in the NCBI Gene Expression Omnibus and will be made accessible upon article acceptance.

\section{References}

1. Pennisi, E. Searching for the Genome's Second Code. Science 306, 632-635 (2004). 
2. Wallace, J. G. et al. Association Mapping across Numerous Traits Reveals Patterns of Functional Variation in Maize. PLOS Genet. 10, e1004845 (2014).

3. Tong, H. \& Chu, C. Functional Specificities of Brassinosteroid and Potential Utilization for Crop Improvement. Trends Plant Sci. 23, 1016-1028 (2018).

4. Yu, M.-H., Zhao, Z.-Z. \& He, J.-X. Brassinosteroid Signaling in Plant-Microbe Interactions. Int. J. Mol. Sci. 19, 4091 (2018).

5. Hartwig, T. et al. Brassinosteroid control of sex determination in maize. Proc. Natl. Acad. Sci. 108, 19814-19819 (2011).

6. Sun, Y. et al. Integration of Brassinosteroid Signal Transduction with the Transcription Network for Plant Growth Regulation in Arabidopsis. Dev. Cell 19, 765-777 (2010).

7. Sun, F. et al. Maize transcription factor ZmBES1/BZR1-5 positively regulates kernel size. J. Exp. Bot. (2020) doi:10.1093/jxb/eraa544.

8. Zhang, X., Guo, W., Du, D., Pu, L. \& Zhang, C. Overexpression of a maize BR transcription factor ZmBZR1 in Arabidopsis enlarges organ and seed size of the transgenic plants. Plant Sci. Int. J. Exp. Plant Biol. 292, 110378 (2020).

9. Best, N. B. et al. nana plant2 Encodes a Maize Ortholog of the Arabidopsis Brassinosteroid Biosynthesis Gene DWARF1, Identifying Developmental Interactions between Brassinosteroids and Gibberellins. Plant Physiol. 171, 2633-2647 (2016).

10. Kir, G. et al. RNA Interference Knockdown of BRASSINOSTEROID INSENSITIVE1 in Maize Reveals Novel Functions for Brassinosteroid Signaling in Controlling Plant Architecture1[OPEN]. Plant Physiol. 169, 826-839 (2015).

11. Hartwig, T. et al. Propiconazole Is a Specific and Accessible Brassinosteroid (BR) Biosynthesis Inhibitor for Arabidopsis and Maize. PLOS ONE 7, e36625 (2012).

12. Best, N. B., Johal, G. \& Dilkes, B. P. Phytohormone inhibitor treatments phenocopy 
brassinosteroid-gibberellin dwarf mutant interactions in maize. Plant Direct 1, (2017).

13. Ryu, H. et al. Nucleocytoplasmic Shuttling of BZR1 Mediated by Phosphorylation Is Essential in Arabidopsis Brassinosteroid Signaling. Plant Cell 19, 2749-2762 (2007).

14. Wang, Z.-Y. et al. Nuclear-Localized BZR1 Mediates Brassinosteroid-Induced Growth and Feedback Suppression of Brassinosteroid Biosynthesis. Dev. Cell 2, 505-513 (2002).

15. Bai, M.-Y. et al. Functions of OsBZR1 and 14-3-3 proteins in brassinosteroid signaling in rice.

Proc. Natl. Acad. Sci. U. S. A. 104, 13839-13844 (2007).

16. Wang, Y. et al. BZR1 Mediates Brassinosteroid-Induced Autophagy and Nitrogen Starvation in Tomato. Plant Physiol. 179, 671-685 (2019).

17. He, J.-X. et al. BZR1 Is a Transcriptional Repressor with Dual Roles in Brassinosteroid Homeostasis and Growth Responses. Science 307, 1634-1638 (2005).

18. Oh, E. et al. Cell elongation is regulated through a central circuit of interacting transcription factors in the Arabidopsis hypocotyl. elife 3, e03031 (2014).

19. Rodgers-Melnick, E., Vera, D. L., Bass, H. W. \& Buckler, E. S. Open chromatin reveals the functional maize genome. Proc. Natl. Acad. Sci. U. S. A. 113, E3177-3184 (2016).

20. Oh, E., Zhu, J.-Y. \& Wang, Z.-Y. Interaction between BZR1 and PIF4 integrates brassinosteroid and environmental responses. Nat. Cell Biol. 14, 802-809 (2012).

21. Yin, Y. et al. A New Class of Transcription Factors Mediates Brassinosteroid-Regulated Gene Expression in Arabidopsis. Cell 120, 249-259 (2005).

22. Regulski, M. et al. The maize methylome influences mRNA splice sites and reveals widespread paramutation-like switches guided by small RNA. Genome Res. 23, 1651-1662 (2013).

23. Zhao, X., Chai, Y. \& Liu, B. Epigenetic inheritance and variation of DNA methylation level and pattern in maize intra-specific hybrids. Plant Sci. 172, 930-938 (2007).

24. Gent, J. I. et al. Accessible DNA and Relative Depletion of H3K9me2 at Maize Loci Undergoing 
RNA-Directed DNA Methylation[W][OPEN]. Plant Cell 26, 4903-4917 (2014).

25. Baldauf, J. A., Marcon, C., Paschold, A. \& Hochholdinger, F. Nonsyntenic Genes Drive TissueSpecific Dynamics of Differential, Nonadditive, and Allelic Expression Patterns in Maize Hybrids. Plant Physiol. 171, 1144-1155 (2016).

26. Zhang, A. et al. Nitric Oxide Mediates Brassinosteroid-Induced ABA Biosynthesis Involved in Oxidative Stress Tolerance in Maize Leaves. Plant Cell Physiol. 52, 181-192 (2011).

27. Liu, T. et al. Expression and functional analysis of ZmDWF4, an ortholog of Arabidopsis DWF4 from maize (Zea mays L.). Plant Cell Rep. 26, 2091-2099 (2007).

28. Marand, A. P., Zhang, T., Zhu, B. \& Jiang, J. Towards genome-wide prediction and characterization of enhancers in plants. Biochim. Biophys. Acta BBA - Gene Regul. Mech. 1860, 131139 (2017).

29. Oka, R. et al. Genome-wide mapping of transcriptional enhancer candidates using DNA and chromatin features in maize. Genome Biol. 18, 137 (2017).

30. Pressoir, G. et al. Natural variation in maize architecture is mediated by allelic differences at the PINOID co-ortholog barren inflorescence2. Plant J. 58, 618-628 (2009).

31. Kump, K. L., Holland, J. B., Jung, M. T., Wolters, P. \& Balint-Kurti, P. J. Joint Analysis of NearIsogenic and Recombinant Inbred Line Populations Yields Precise Positional Estimates for Quantitative Trait Loci. Plant Genome 3, (2010).

32. Nolan, T. M., Vukašinović, N., Liu, D., Russinova, E. \& Yin, Y. Brassinosteroids: Multidimensional Regulators of Plant Growth, Development, and Stress Responses. Plant Cell 32, 295-318 (2020). 


\section{Material and Methods}

\section{Plant material and growth conditions}

Construction of the ZmBES1/BZR1-YFP transgenic line was previously described ${ }^{1}$ and obtained in Hill background from A.W. Silvester. The ZmBZR1-YFP line was further crossed six times with B73, using B73 as mother to avoid contamination. Wild-type and ZmBES1/BZR1-YFP, and BR deficient mutant (brd1) plants were grown side by side in greenhouses, under long-day conditions (16h day/8h night, $28-30^{\circ} \mathrm{C}$ ), and in the 20132016 Carnegie Institution for Science summer fields (Stanford, California, USA).

\section{B73 ChIP-seq; B73 / Mo17 HASCh-seq, and ChIP-qPCR}

ZmBZR1-YFP/B73, Mo17 inbred as well as ZmBZR1-YFP/B73xMo17 and Mo17xZmBZR1-YFP/B73 F1 hybrid plants were grown under greenhouse conditions for 26 days. The oldest 2 leaves were removed and $2 \mathrm{~cm}$ of meristem enriched tissue was used (Supplementary Fig. 1). Tissues were first treated with $1 \mu \mathrm{M} \mathrm{BL}$ for $4 \mathrm{~h}$ at room temperature $(\mathrm{RT})$ in water. After BR treatment, tissues were cross-linked with $2 \%$ formaldehyde for 10 min under vacuum with 5 min incubation after release. Tissues were ground to a fine powder in liquid nitrogen and nuclei extraction was performed as described in ${ }^{2}$. Nuclear extracts were sonicated using a Branson 250 Sonifier (2x 4 min on time, $20 \mathrm{sec}$. on/off cycle with 10 min rest between repeats, $20 \%$ amplitude), and after removing an Input aliquot, incubated for $2 \mathrm{~h}$ with $10 \mathrm{ug}$ polyclonal Anti-GFP antibody ${ }^{2}$ (Supplementary Fig. 1). Protein-DNA complexes were captured on Dynabeads-Protein G (Life Technologies, \#10003D) and the beads were washed with low-salt buffer (50 mM 
Tris- $\mathrm{HCl}$ at $\mathrm{pH}$ 8.0, $2 \mathrm{mM}$ EDTA, $150 \mathrm{mM} \mathrm{NaCl}, 0.5 \%$ Triton X-100), with high-salt buffer (50 mM Tris- $\mathrm{HCl}$ at pH 8.0, $2 \mathrm{mM}$ EDTA, $500 \mathrm{mM} \mathrm{NaCl}, 0.5 \%$ Triton X-100), with LiCl buffer $(10 \mathrm{mM}$ Tris- $\mathrm{HCl}$ at $\mathrm{pH}$ 8.0, $1 \mathrm{mM}$ EDTA, $0.25 \mathrm{M} \mathrm{LiCl}, 0.5 \% \mathrm{NP}-40,0.5 \%$ deoxycholate) and twice with TE buffer $(10 \mathrm{mM}$ Tris- $\mathrm{HCl}$ at $\mathrm{pH} 8.0,1 \mathrm{mM}$ EDTA) and eluted with elution buffer ( $1 \% \mathrm{SDS}, 0.1 \mathrm{M} \mathrm{NaHCO})$ at $65^{\circ} \mathrm{C}$ overnight. After a column purification, (Quiagen, PCR purification kit), ChIP-seq libraries were generated using the Ultra-II kit (NEB), following manufacture recommendations using $10 \mathrm{ng}$ per sample as starting material. ChIP qPCR was performed using the Bioline SensiFAST SYBR Kit following manufacturer recommendations on a Roche LightCylcer 480 at $63^{\circ} \mathrm{C}$ annealing temperature. Primers used for the analysis are listed in Table S11.

\section{ChIP-seq data analysis}

Quality filtered ChIP-seq reads were aligned to the B73 AGPv4 genome using bwa-mem (v. 0.7.16a) $)^{3}$ with default parameters, followed by removal of PCR duplicates using samtools (v. 1.3.1.) $)^{4}$. To determine BZR1 binding peaks, IP and negative control samples, after normalization for read depth, were analyzed using the GEM package (v. 3.0) ${ }^{5}$ (using parameters: --fold 5, --k_min 5, --k_max 8). After samples were analyzed individually, peaks reproducible in all 3 replicas, using a 400 bp window (+/- 200 bp around each peak) were determined using $R$ (v. 3.3.2) and considered high confidence peaks. 


\section{HASCh-seq data analysis}

Mitigating potential biases are critical steps in order to accurately analyze allele specific binding. For HASCh-seq, we first tried to address mapping bias using an enhanced reference approach previously described ${ }^{6}$. After generating a pseudo Mo17 reference genome by integrating all homozygous B73/Mo17 HapMap 3.2.1 SNPs into the B73 AGPv4 genome ( $\mathrm{R}$ v.3.3.2), we mapped quality filtered paired-end reads against the B73 and pseudo Mo17 reference genomes with bwa-mem (v. 0.7.16a) using default parameters. Next, we merged the corresponding aligned reads, retaining uniquely or higher quality mapping reads to either B73 or Mo17, or a random read in case of equal mapping quality using the merge-sam script ${ }^{7}$. Finally, we filtered for uniquely mapped reads (q13), and removed potential PCR duplicates (retaining a random read, instead higher mapping quality to avoid B73 bias). Overlapping mate reads were trimmed to avoid duplicated counting using the clip_overlap script (https://genome.sph.umich.edu/wiki/BamUtil: clipOverlap). Bias corrected mapping files were used to determine both BZR1 binding peaks using the GEM pipeline described above, as well as ASBs. To determine high confidence peaks, samples were analyzed individually and peaks reproducible in all 6 replicates, using a 400 bp window (+/- 200 bp around each peak) were retained.

To identify regions with differential TF binding, we first merged bias-corrected bam files for IP or Input replicates and determined allele frequencies for all homozygotes HapMap 3.2.1. B73/Mo17 SNPs $(6,685,773)$ determined using ASEReadCounter (GATK 3.6). ZmBZR1-YFP ASBs were determined using custom R (v. 3.3.2) scripts. In order to accurately access allele frequencies of all homozygous SNPs, we set a minimum read 
coverage cutoff of $\geq 50$ reads $(n=162,398)$. Next, to reduced potential artifacts due to false positive B73/Mo17 SNPs, base calling errors in the B73 AGPv4 reference genome and sequencing errors, we removed SNPs with a bias $\geq 90 \%$ to either parent, if both the B73 and Mo17 allele could not be verified in the input data ( $n=160,755$ SNPs). To further reduce potential background or artificial bias effects, we blacklisted genomic regions (chr. 3: $186,240,476-210,080,072$; chr10: $2,965,337-4124958)$ with a systematic bias in the input data.

Of the remaining 151,073 SNPs, we determined significant variation of median allele frequency of 0.515 using a binomial test with a p-value cutoff of $\leq 0.001$ adjusted for multiple testing using Bonferroni correction ( $n=19,601$ SNPs). To focus on ASBs with potential biological relevance, we further restricted ASBs to those located in highconfidence BZR1-binding peaks reproducible in all 6 biological replicates. While TFs usually bind small DNA regions of $\sim 10 \mathrm{bp}^{8}$, we used 75 bp paired-end sequencing with an average insert size of $\sim 200$ bp achieved after sonication. Therefore, SNPs in close proximity with causative polymorphisms will show biased allele frequency due to linkage disequilibrium (LD). To address this, we identified SNPs with significant bias within a $150 \mathrm{bp}$ rolling window of each other and defined the lead SNP of ASBs by the lowest $p$ value, integrating both read depth and allele frequency bias.

\section{Control background SNP (bgSNP) sampling}

Functional GWAS variants have been shown to be significantly enriched in gene proximal regions $^{9}$. Therefore, control background SNPs (bgSNPs) were proportionally sampled 
(excluding ASBs) per chromosome and genomic location (i.e. 5 - 1kb upstream, $1 \mathrm{~kb}$ upstream - TSS, 5'UTR, exon, intron, 3'UTR, TTS - 1 kb downstream, intergenic) to match the genomic distribution of the ASBs dataset. Additionally, we checked that ASBs and bgSNPs showed a similar minor allele-frequency (Supplementary Fig. 3). In total, 168950 bgSNPs were sampled, yielding approximately 50 times as many background SNPs per genome location, compared to the number of ASBs within each location. Sequencing data that support the finding of this study have been deposited in the NCBI SRA database and will be made available upon acceptance of the manuscript.

\section{Fluorescence imaging}

Heterozygote BZR1-YFP plants were grown in the dark at RT for 10 days in vermiculite with and without $10 \mu \mathrm{M}$ PPZ. Prior to imaging, $1 \mathrm{~cm}$ root tip segments were removed from the mock and PPZ-treated seedlings. The root tips of PPZ-treated plants were treated for 15 min with $10 \mu \mathrm{M} \mathrm{PPZ}$ with or without $1 \mu \mathrm{M}$ 24epi-BL.

\section{RNA extraction, RNA sequencing and differential expression analysis}

BR deficient brd1 mutant siblings were grown in soil under greenhouse conditions for 26 days as described above. The oldest 2 leaves were removed and $2 \mathrm{~cm}$ of meristem enriched tissue (Supplementary Fig. 1) were placed in $1 \mu \mathrm{M} \mathrm{BL}$ for $4 \mathrm{~h}$ at room temperature (RT) in water. Total RNA was isolated using acidic phenol extraction as described previously ${ }^{10}$. Purification of poly-adenylated mRNA using oligo(dT) beads, construction of barcoded libraries, and sequencing using Illumina HiSeq 4500 technology 
(75 bp paired-end reads) were performed by Novogene Co. using manufacturer's Illumina recommendations. Trimmed and QC (Seqpurge v. 2019-02-11) filtered sequence reads were mapped to the B73 AGPv4 genome using STAR (v. 2.54) ${ }^{11}$ in twopass mode (with parameters: --outFilterScoreMinOverLread 0.3, --outFilterMatchNminOverLread 0.3, -outSAMstrandField intronMotif, --outFilterType BySJout, --outFilterIntronMotifs RemoveNoncanonical, --quantMode TranscriptomeSAM GeneCounts). Unique reads were filtered by mapping quality (q20) and PCR duplicates removed using Samtools (v. 1.3.1). Gene expression was analyzed in $R$ (v. 3.4.1) using the DEseq2 software (v. 1.16.1 $)^{12}$. Genes were defined as differentially expressed by a 1.5 -fold expression difference with a $p$ value, adjusted for multiple testing, of $<0.05$. Accession number for the RNA-seq data in the Gene Expression Omnibus database will be available upon paper acceptance. For the analysis of allele-specific gene regulation a previously published RNA-seq data set from B73xMo17 F1 hybrids was used (Baldauf et al., 2016).

\section{Genomic feature profiling of ASBs (Methylation, Motifs and DNAsel, Enhancers)}

Methylation levels for $\mathrm{CG}, \mathrm{CHG}$, and $\mathrm{CHH}$ for $\mathrm{B} 73$ and $\mathrm{Mo17}$ were extracted from Regulski et al. $2013^{13}$. Methylation frequency versus distance (up to $+/-2 \mathrm{kbp}$ ) around each ASB were averaged over 20bp bins, and visualized by regions bound by BZR1 with either high or low affinity levels depending on the inbred line. For B73, high and low affinity bound regions were defined by a post frequency of $>=0.85$ or $<=0.15$, respectively and oppositely for Mo17 by a post frequency $<=0.15$ and $>=0.85$ ), respectively. 
For local motif enrichment analysis (Fig. $2 \mathrm{E}$ ), we extracted $+/-50 \mathrm{bp}$ of the high affinity BZR1 bound allele surrounding ASBs. The MEME CentriMo suite (v. 5.2.0) was used to determine the local distribution along the $101 \mathrm{bp}$ fragments for the canonical BZR1 motifs BRRE (CGTG[T/C]G, C[G/A]CACG) or G-box (CACGTG) and a control motif, with SBP ("GTACGG", "CCGTAC") ${ }^{14}$, with a similar GC content.

To identify ASBs which may be explained by motif variation (Fig. 2h), we extracted the +/- $5 \mathrm{bp}$ of the high affinity BZR1 bound allele surrounding ASBs. Using R we scanned those $11 \mathrm{bp}$ fragments for canonical BRRE (CGTG[T/C]G, C[G/A]CACG), allowing a single base pair mismatch outside the core motif (CGTG), or G-box (CACGTG) motifs and determined ASBs where the SNP changed a BRRE or G-box motif into an altered (non BRRE or G-box) motif.

To identify ASBs which overlapped with significant variation in either $\mathrm{CG}, \mathrm{CHG}$, or $\mathrm{CHH}$ methylation between B73 and Mo17, we first, per ASB, assigned averaged methylation levels of Mo17 and B73 methylation levels (separately for the CG, $\mathrm{CHG}$, or $\mathrm{CHH}$ methylation datasets) within a given window of $+/-40$ bp around the ASB position. Differentially methylated alleles were defined as described previously ${ }^{13}$. Accordingly, we identified ASBs as overlapping with differentially methylated regions if in the B73 or Mo17 methylation datasets, the methylation level of one allele would be $>=70 \%$ while the level of the corresponding allele was $<=10 \%$.

Putative B73 enhancer (Fig. 4a) regions were extracted from Oka et al. 2017 ${ }^{15}$. To determine potential enrichments, +/- $10 \mathrm{kbps}$ surrounding enhancer regions were intersected with ASBs and bgSNPs. 


\section{GWAS enrichment, Kinship matrices and variance components analysis}

We tested association of ASBs with the curated 4041 significant GWAS hits for 41 different phenotypes of the NAM population ${ }^{9}$. Per trait we performed an enrichment between the ASBs compared to the control bgSNPs (with the same average genomic distribution and minor allele frequency (Supplementary Fig. 4)). GWAS hits were counted if they were located within 2 kbps of ABSs or bgSNPs and the subsequent enrichment analysis was based on a hyper-geometric test.

We estimated the variance components explained by different ASB SNP subsets and the remaining SNPs using the maize Nested Association Mapping (NAM) population ${ }^{16}$. To conduct the analysis, we downloaded the phenotypic data (/iplant/home/glaubitz/RareAlleles/genomeAnnos/VCAP/phenotypes/

NAM/familyCorrected), consisting of Best Linear Unbiased Predictors (BLUPs) for different traits $^{9}$, and the imputed genotypic data (/iplant/home/glaubitz/RareAlleles/genomeAnnos/VCAP/genotypes/NAM/namrils_projec ted_hmp31_MAF02mnCnt2500.hmp.txt.gz) ${ }^{17}$ from CyVerse database as described in Panzea (www.panzea.org). In the analysis, we mapped ASB SNPs and randomly sampled bgSNPs that shared the similar genomic patterns to upstream 5kb-TSS, within the CDS, TTS-5Kb downstream of genes as well as intergenic regions (see control bgSNP section for details). For each SNP subset, we calculated an additive kinship matrix using the variance component annotation pipeline (VCAP) implemented in TASSEL5 ${ }^{18}$. We then fed these kinship matrices along with the NAM phenotypic data to estimate the variance components explained by the ASB subsets, using a Residual Maximum Likelihood (REML) method implemented in LDAK $^{19}$. 


\section{References Material and Methods}

1. Kir, G. et al. RNA Interference Knockdown of BRASSINOSTEROID INSENSITIVE1 in Maize Reveals Novel Functions for Brassinosteroid Signaling in Controlling Plant Architecture1[OPEN]. Plant Physiol. 169, 826-839 (2015).

2. Oh, E. et al. Cell elongation is regulated through a central circuit of interacting transcription factors in the Arabidopsis hypocotyl. eLife 3, e03031 (2014).

3. Li, H. \& Durbin, R. Fast and accurate short read alignment with Burrows-Wheeler transform. Bioinforma. Oxf. Engl. 25, 1754-1760 (2009).

4. Li, H. et al. The Sequence Alignment/Map format and SAMtools. Bioinforma. Oxf. Engl. 25, 2078-2079 (2009).

5. Guo, Y., Mahony, S. \& Gifford, D. K. High Resolution Genome Wide Binding Event Finding and Motif Discovery Reveals Transcription Factor Spatial Binding Constraints. PLOS Comput. Biol. 8, e1002638 (2012).

6. Huang, S., Holt, J., Kao, C.-Y., McMillan, L. \& Wang, W. A novel multi-alignment pipeline for high-throughput sequencing data. Database J. Biol. Databases Curation 2014, (2014). 7. Castel, S. E., Levy-Moonshine, A., Mohammadi, P., Banks, E. \& Lappalainen, T. Tools and best practices for data processing in allelic expression analysis. Genome Biol. 16, 195 (2015).

8. Stewart, A. J., Hannenhalli, S. \& Plotkin, J. B. Why Transcription Factor Binding Sites Are Ten Nucleotides Long. Genetics 192, 973-985 (2012).

9. Wallace, J. G. et al. Association Mapping across Numerous Traits Reveals Patterns of Functional Variation in Maize. PLOS Genet. 10, e1004845 (2014).

10. Eggermont, K., Goderis, I. J. \& Broekaert, W. F. High-throughput RNA extraction from plant samples based on homogenisation by reciprocal shaking in the presence of a mixture of sand and glass beads. Plant Mol. Biol. Report. 14, 273-279 (1996).

11. Dobin, A. et al. STAR: ultrafast universal RNA-seq aligner. Bioinformatics 29, 15-21 
(2013).

12. Love, M. I., Huber, W. \& Anders, S. Moderated estimation of fold change and dispersion for RNA-seq data with DESeq2. Genome Biol. 15, 550 (2014).

13. Regulski, M. et al. The maize methylome influences mRNA splice sites and reveals widespread paramutation-like switches guided by small RNA. Genome Res. 23, 1651-1662 (2013).

14. O'Malley, R. C. et al. Cistrome and Epicistrome Features Shape the Regulatory DNA Landscape. Cell 165, 1280-1292 (2016).

15. Oka, R. et al. Genome-wide mapping of transcriptional enhancer candidates using DNA and chromatin features in maize. Genome Biol. 18, 137 (2017).

16. Yu, J., Holland, J. B., McMullen, M. D. \& Buckler, E. S. Genetic Design and Statistical Power of Nested Association Mapping in Maize. Genetics 178, 539-551 (2008).

17. Bukowski, R. et al. Construction of the third-generation Zea mays haplotype map. GigaScience 7, (2018).

18. Bradbury, P. J. et al. TASSEL: software for association mapping of complex traits in diverse samples. Bioinformatics 23, 2633-2635 (2007).

19. Speed, D., Hemani, G., Johnson, M. R. \& Balding, D. J. Improved Heritability Estimation from Genome-wide SNPs. Am. J. Hum. Genet. 91, 1011-1021 (2012). 
Figures and legends:

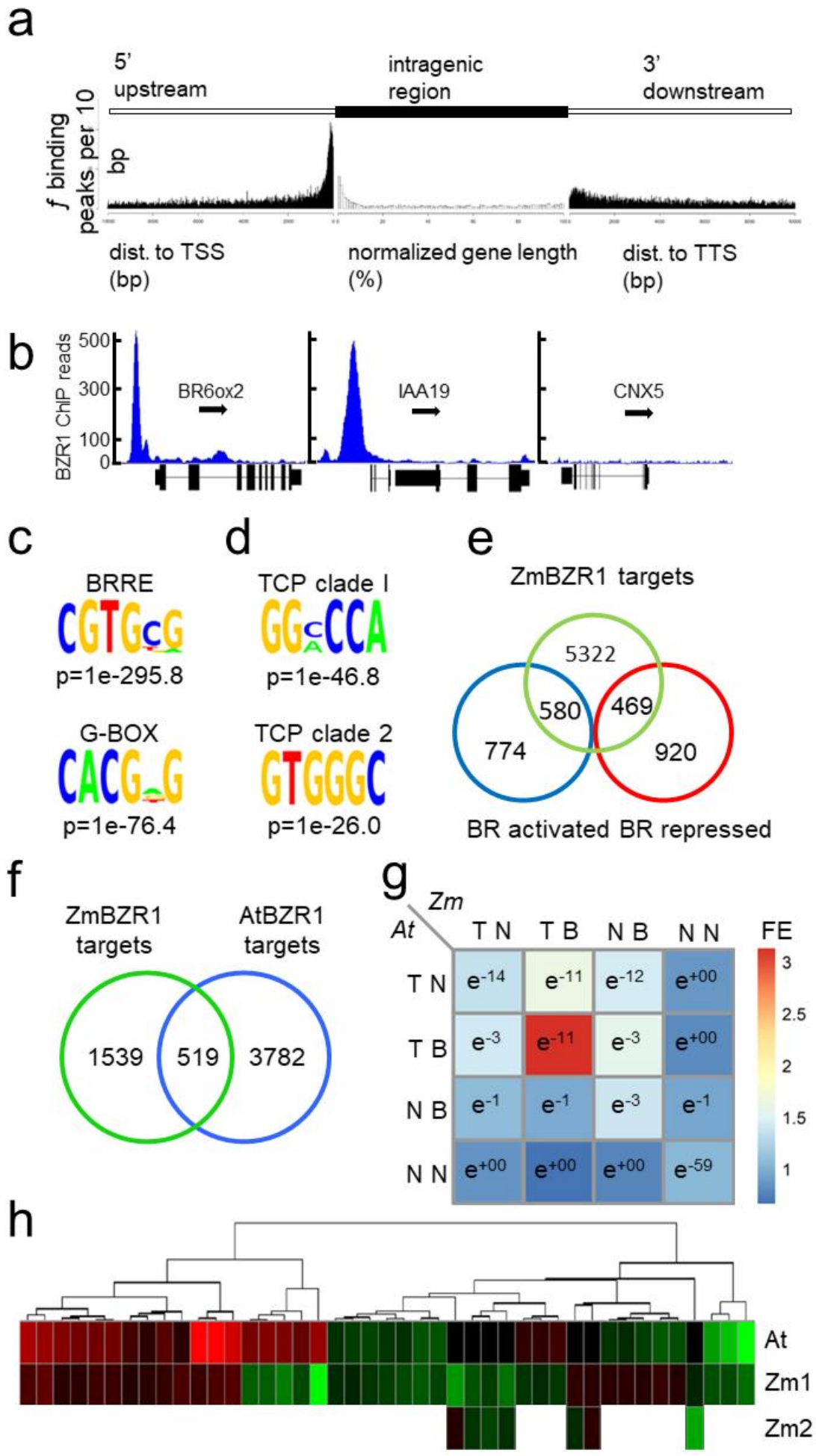

Fig. 1. BZR1 regulatory network in maize and Arabidopsis. B73 ChIP-seq results. a) Distribution of BZR1 binding around transcribed genes. Frequency of BZR1 binding peaks up to $10 \mathrm{~kb}$ up- or downstream of TSS or TTS and intra-genic, respectively. b) ChIP-seq identified BZR1 
binding in proximity to putative targets repressed (BR6ox2), induced (IAA19) or not controlled by BR (CNX5). Blue peaks represent normalized reads mapped to the B73v4 genome. Black arrows indicate direction of transcription. c) and d) Significantly overrepresented ZmBZR1 binding motifs, BRRE (CGTG[C/T]G) and Gbox (CACGTG) (c) as well as BRRE significantly co-localized secondary motifs for TCP TF class I (GG[A/C]CCA) and class II (GTGGGC) determined by GEM (d). e) Direct and indirect targets of BZR1. Shown is the overlap of BZR1 ChIPseq and RNAseq of the BR deficient brd1 mutant +/- BR. f) Conservation of the BZR1 direct targets between Arabidopsis and maize. Overlap of orthologues Arabidopsis and maize BZR targets. g) Overlap of orthologous BR responsive (B) and non-responsive (N) BZR1 target genes $(T)$ and non-target genes (N) in Arabidopsis (At) and Maize (Zm). Color-coding indicates fold enrichment (FE) compared to random expectation (reaching from blue, low enrichment to red, high enrichment), numbers indicate $\mathrm{p}$-values of significance of this enrichment assuming a hypergeometric distribution. h) Heatmap of direct orthologues target genes of BZR1 in Arabidopsis and maize induced (green) or repressed (red) by BR. Overall, $65 \%$ of $1: 1$ orthologs and $72 \%$ of $1: 2$ copy orthologs of direct BZR1 targets between Arabidopsis and Maize, respectively, showed the same direction of $B R$ regulation. 
a

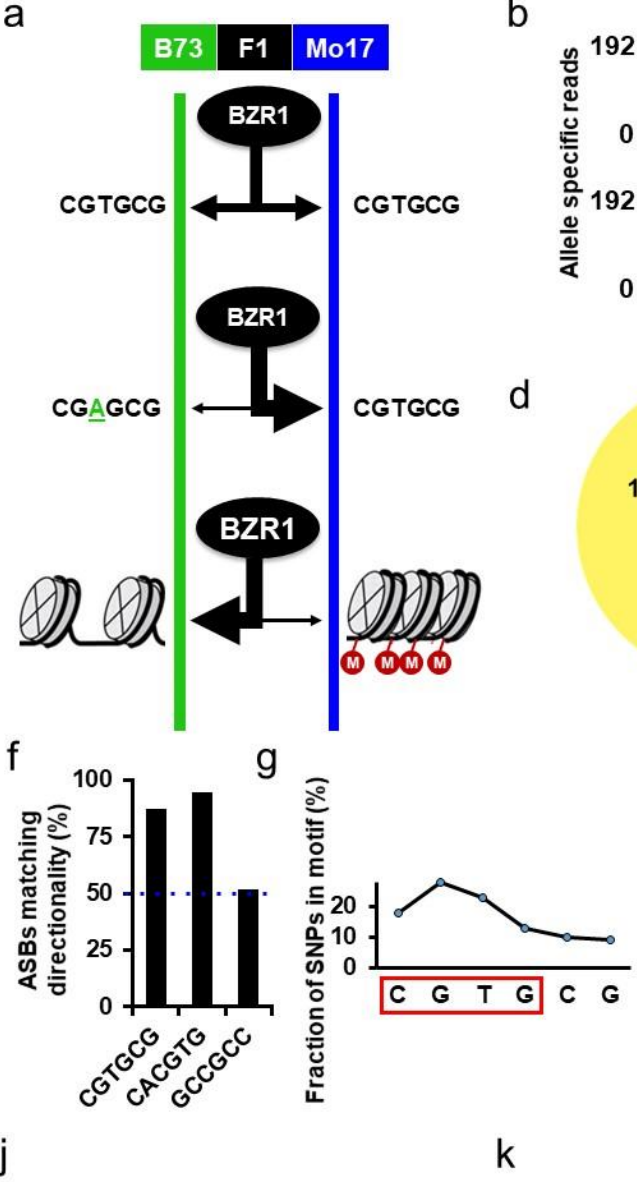

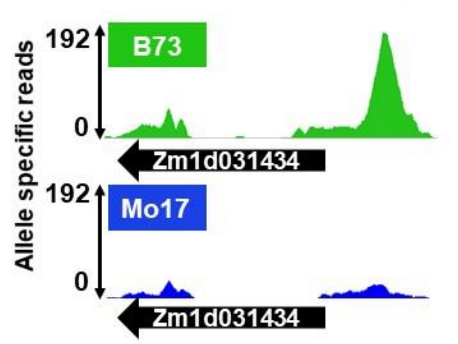

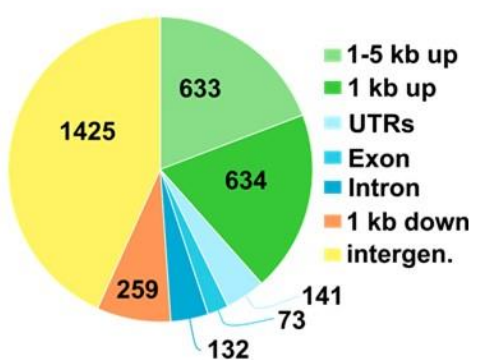

h

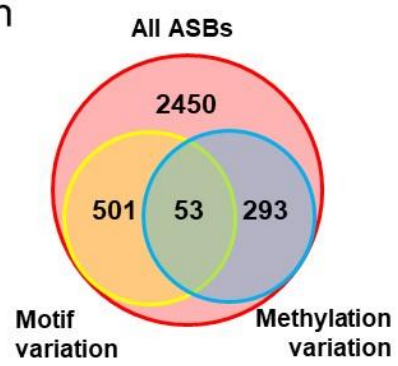

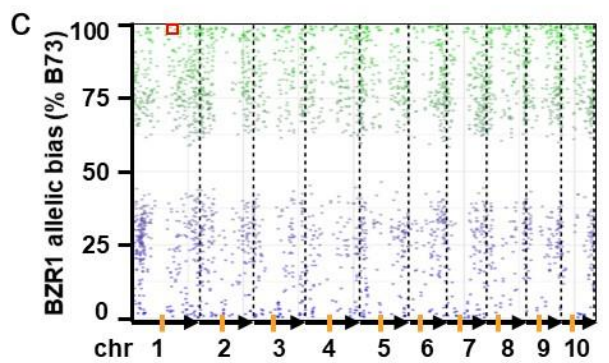

e
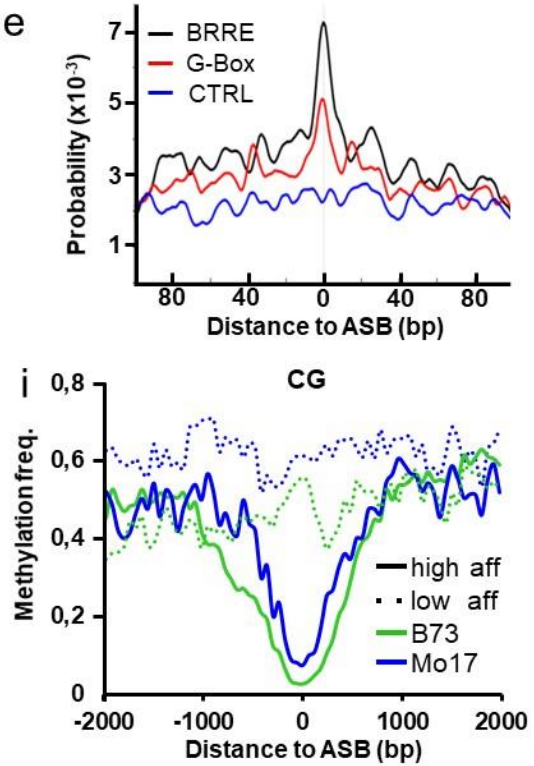

CG
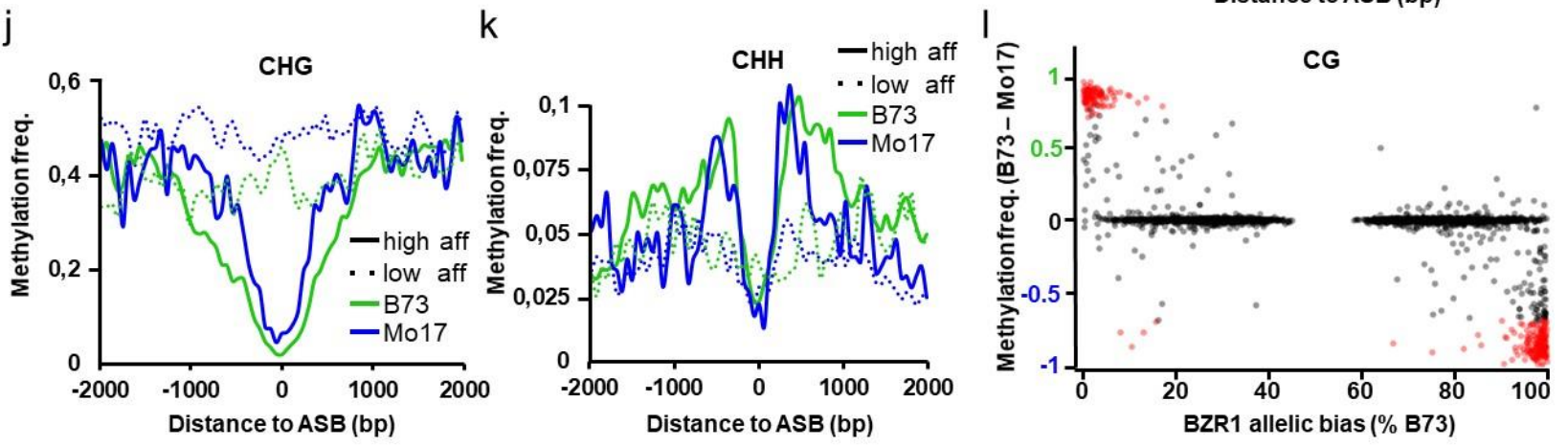

Fig. 2. Variation in DNA sequence and methylation underlie differential BZR1 binding. a) Schematics of HASCh-seq approach and possible causes for allele-specific binding events. Chromatin-IP is performed in F1 hybrid plants. From top to bottom, three possible scenarios for TF binding to the parental genomes (green and blue) are depicted, where binding strength is represented by the line width of the black arrows: when no alterations in motif and chromatin structure occurs, binding is expected to be equal. Lower binding is expected If the binding motif is altered by a SNP or epigenetic features like DNA methylation for one of the alleles. b) An example of allele-specific binding of ZmBZR1 to target gene Zm00001d031434. BZR1 bound target reads that uniquely map to either B73 (green) or Mo17 (blue) are shown. c) Allelic and spatial distribution of allele-specific BZR1 binding sites with significant bias towards B73 (green) or Mo17 (blue) along the maize B73 reference genome. Allelic bias is expressed as percentage of B73 read counts. Chromosome borders and length are depicted by dashed lines and arrows at the bottom, respectively. Centromere locations are indicated by orange rectangles. A red box on 
chromosome 1 marks the ASB upstream of Zm00001d031434 displayed in Fig. 2b. d) Genomic distribution of ASBs. 3297 ASBs were classified according to their location relative to genes. In case of two genes in the proximity of an $A S B$, the priority given was exon>intron $>U T R>1 \mathrm{~kb}$ upstream $>1 \mathrm{~kb}$ downstream $>1-5 \mathrm{~kb}$ upstream. e) Frequency of BRRE (CGTG[C/T]G), G-Box (CACGTG), and the control motif GTACGG (SBP-box ${ }^{12}$ ) around ASBs of the alleles with higher BZR1 binding. f) Fraction of ASBs overlapping with motifs, for which the allele with canonical BRRE, G-Box or a control motif GCCGCC (GCC-box ${ }^{12}$ ), showed higher ZmBZR1 affinity. Both BR-related BRRE and G-box motifs, but not the control motif, diverge significantly $(p<0.001$, Fisher's exact test) from the expected $50 \%$ random distribution. g) Distribution of lead SNPs of ASBs within motifs. Among ASBs which overlapped with BRRE motif, SNPs were enriched in the core CG bases. $h$ ) ASBs affecting BZR1 motifs and/or overlapping with differentially methylated (CpG, CHG or $\mathrm{CHH}$ ) sites between B73 and Mo17. i)-k) Average $\mathrm{CpG}$ (h), $\mathrm{CHG}$ (i) and $\mathrm{CHH}(\mathrm{j})$ methylation frequency in B73 (green) and Mo17 (blue) over ASB loci with a least 85\% binding bias towards B73 or Mo17. High affinity (-) and low affinity (‥) alleles are considered separately for each genotype. I) Correlation of CG methylation with allele-specific BZR1 binding. Average CG methylation of the B73 - Mo17 allele of the 40bp surrounding each ASB are plotted against the allelic bias (expressed in percentage of B73 read counts). Differential CG methylation is indicated by red dots. 

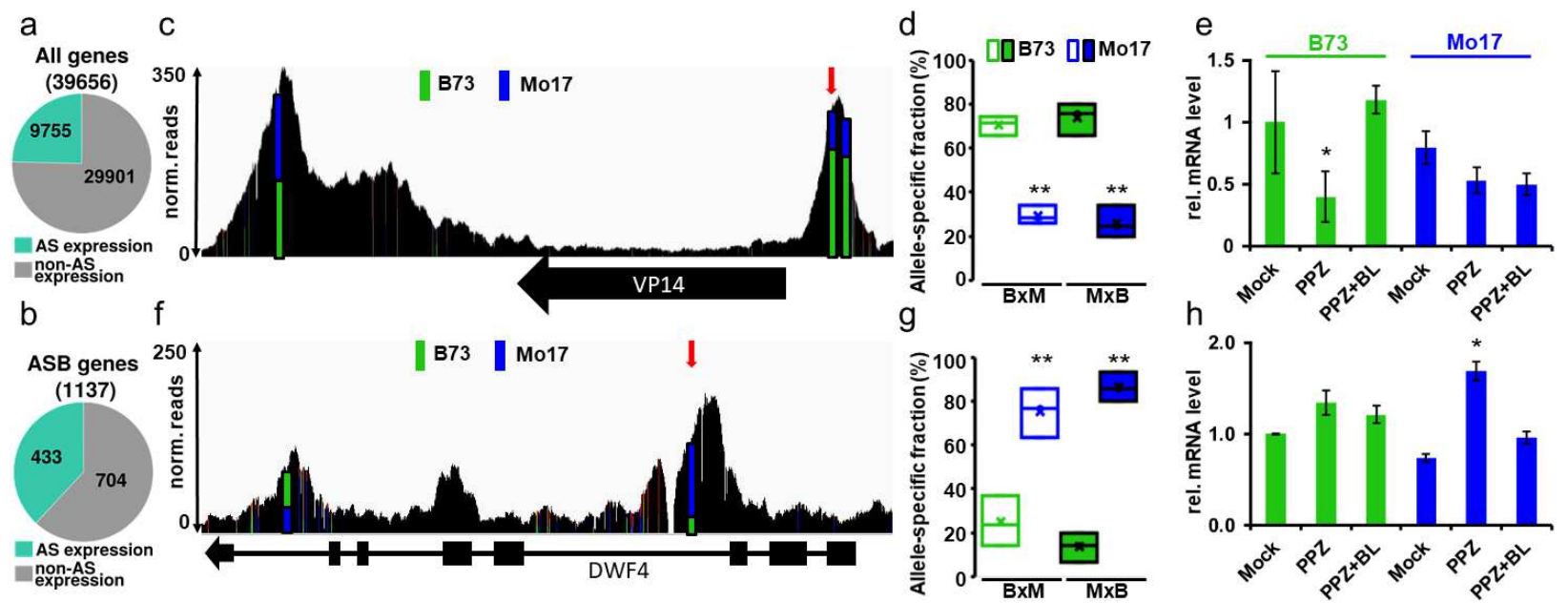

Fig. 3. ASBs correlate with allele-specific mRNA abundance. a) Fraction of all Maize genes displaying allele-specific mRNA abundance between B73 and Mo17. b) Fraction of ASBassociated ZmBZR1-target genes displaying allele-specific mRNA abundance between B73 and Mo17. c) and f) Cumulative HASCh-seq signal and allelic read distribution of ASBs near DWF4 and VP14. Colored bars in the HASCh-seq tracks depict the allelic distribution at SNPs. d) and g) Box plots of allelic reads at two selected ASBs (red arrows in panels $\mathrm{c}$ and $\mathrm{f}$, respectively) from the three replicates HASCh-seq for B73xMo17 (BxM) and Mo17xB73 (MxB) F1 hybrids. e) and h) Transcript levels of the BR-induced VP14 (e) and BR-repressed DWF4 (h) measured by qRTPCR in B73 and Mo17 plants treated with mock, BL inhibitor PPZ, or PPZ plus BL for $4 \mathrm{~h} .{ }^{* *} \mathrm{p}<0.01$, ${ }^{*} \mathrm{p}<0.05$. 
a

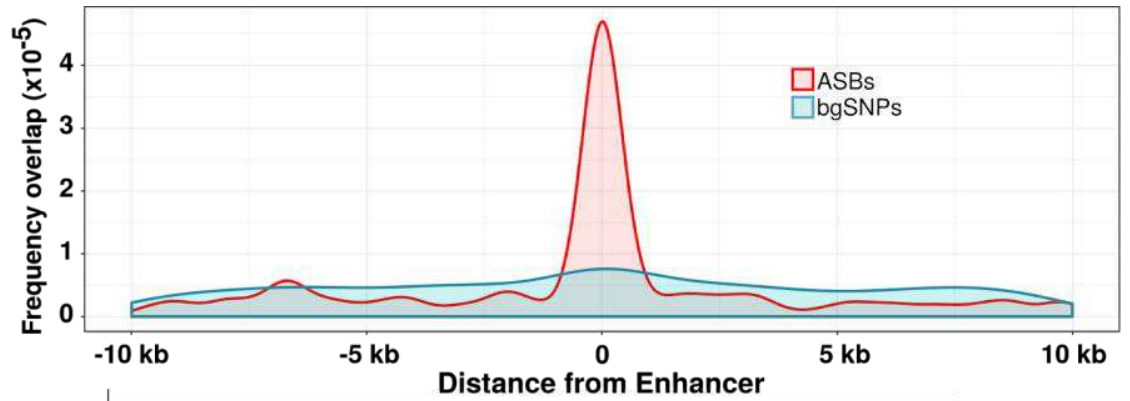

b
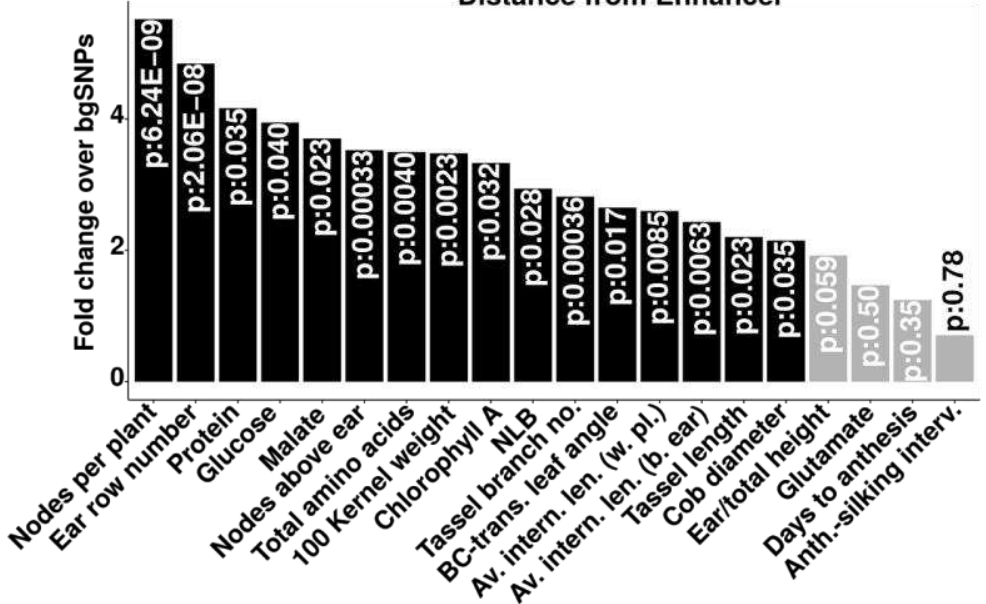

C

100 Kernel weight Leaf width

Cob diameter

Tassel branch number

Anthesis-silking interval

Southern leaf blight

Noders per plant

Days to silk

Ear row number

Tassel length

Northern leaf blight

Nodes above ear.

Nodes to ear

Height/day (until flowering).

Av. intern. len. (whole pl.)

Days to anthesis

Plant height

Ear height

Leaf length

Height above ear.

Av. intern. len. (above ear).

Ear height/total heigh

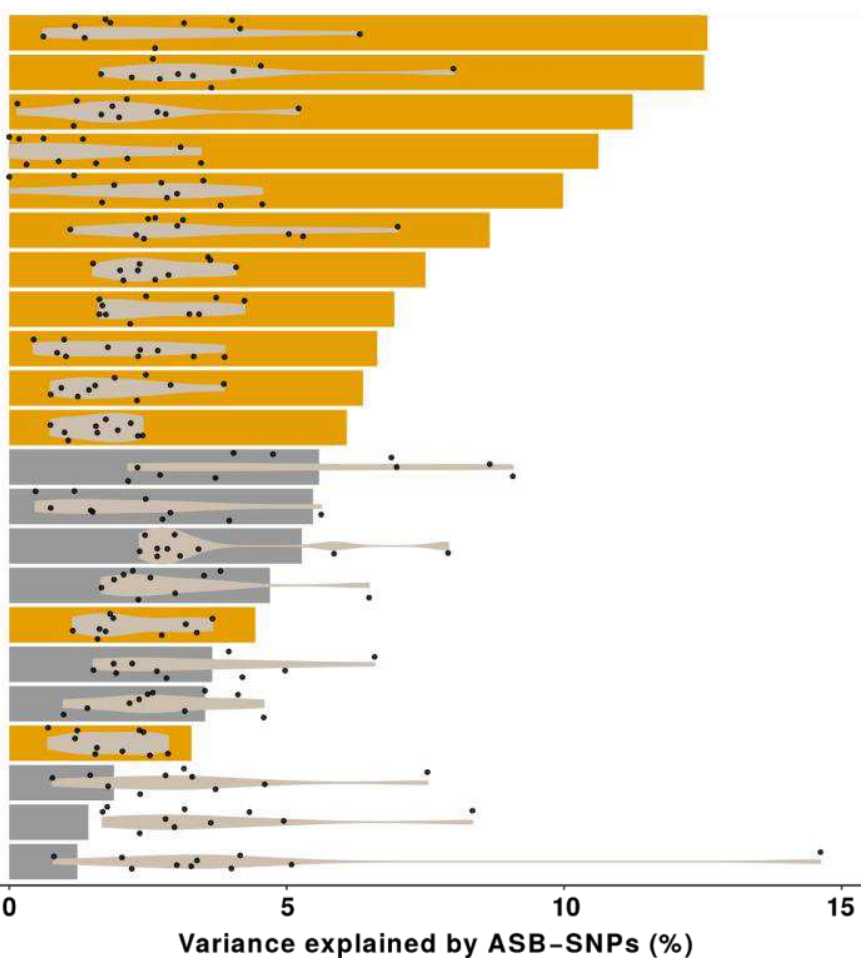

Fig. 4. ASBs of ZmBZR1 are linked to growth and disease related traits. a) ASBs of ZmBZR1 are overrepresented at enhancer sites as compared to randomly selected background (bg) SNPs b) Association of ASBs with the curated 4041 significant GWAS hits for selected phenotypes of the NAM population. Abbreviations: Av: Average; intern: internode; len: length; w. pl.: whole plant; b.: below c) VCAP Variance component analysis results. Variance explained (h2) by the ASBSNP set (bars) and background SNP set (violin plots, derived from the permutation results). 
Orange color in the bars denotes a significantly higher variance explained (h2) by ASBs than expected by chance (one-sided permutation test $<0.1$ ). 


\section{Supplementary Files}

This is a list of supplementary files associated with this preprint. Click to download.

- SupplementalMateriallistlegends.pdf

- FigS1final.jpg

- Figs2final.png

- FigS3.pdf

- Figs4.pdf

- Tables1.txt

- Tables2.txt

- Tables3.txt

- Tables4.txt

- Tables5.txt

- Tables6.txt

- Tables7.txt

- Tables8.txt

- Tables9.txt

- TableS10.xlsx

- TableS11.xlsx 